\title{
Fair rate control for cognitive multi-channel wireless ad hoc networks
}

\author{
Wonyong Yoon ${ }^{1}$ and Wooseong Kim²*
}

\begin{abstract}
Multi-channel wireless ad hoc networks enhance network capacity with the use of available channels in parallel but should strike a balance between maintaining network connectivity and maximizing utilization of different channels. Also, a promising wireless multi-channel ad hoc network should be cognitive to interference from various wireless devices operating on industrial, scientific, and medical bands effectively by scavenging less loaded channels dynamically using periodic spectrum sensing and distributed channel selection algorithm. In this paper, we study cross-layer fair rate control problems for hybrid channel assignment-based multi-channel ad hoc networks in the cognitive context. We first identify two inherent constraints which are specific to multi-channel multi-radio wireless ad hoc networks and primary user interference. We then formulate a fair rate control problem by exploiting the network utility maximization framework and propose a price-based distributed solution. We investigate in detail the convergence property of the distributed solution via simulation on a grid topology. We also show its convergence in adaptation to the change in primary user interference in the cognitive context. We present a performance comparison study with a generic class of existing works in the literature.
\end{abstract}

Keywords: Multi-channel wireless ad hoc network; Fair rate control; Distributed control; Cognitive radio

\section{Introduction}

Multi-channel and multi-radio wireless networks have popularly been studied to increase the network capacity by enriching the freedom of using wireless channels. They lead wireless nodes to avoid effectively intraflow or interflow interferences within wireless networks using multiple orthogonal channels [1-3]. Channel assignment has been a central issue in such multi-channel wireless networks to determine which interface uses which channel in order to maximize the utilization of available channels. Static channel assignment to multiple radios based on network flows leads to a chain effect of channel mobility whenever network topology changes. In contrast, dynamic channel assignment requires an additional rendezvous scheme to meet on the same channel. Hybrid channel assignment however supports flexible and robust connectivities among nodes compared to those static and dynamic channel assignments $[4,5]$ even if it costs channel switching delay. For example, it assigns two different channels to two

*Correspondence: wooseong.kim@samsung.com

2 DMC R\&D Center, Samsung Electronics, Maetan 3-dong 416, Youngtong-gu, Suwon 443-732, South Korea

Full list of author information is available at the end of the article radio interfaces of a mesh node for concurrent operations of receiving and transmitting similar to full-duplex communication. Thus, it enables a transmitting radio interface to switch very rapidly among the receiving channels of neighbor nodes to maintain full connectivity while the other receiving interface stays tuned for the fixed channel to avoid deafness and disconnection problems.

Unlike channel assignment, fair rate control issues in multi-channel wireless networks have been studied in the limited literature. Giannoulis et al. [6] studied congestion control and channel assignment problems in the context of multi-radio wireless mesh networks using a cliquebased interference model and proposed a distributed solution. Tang et al. [7] studied rate control with various fairness objectives in the context of multi-channel wireless mesh networks and proposed a centralized solution. Though both works are targeted for wireless mesh networks, they do not take the different types of nodes, i.e., mesh routers and mesh clients, into their system model. In this paper, we focus on multi-channel wireless ad hoc networks and consider fair rate control issues therein. In the context of ad hoc networks, maintaining the connectivity of networks in the presence of potential change of

\section{至 Springer}

C 2014 Yoon and Kim; licensee Springer. This is an Open Access article distributed under the terms of the Creative Commons Attribution License (http://creativecommons.org/licenses/by/2.0), which permits unrestricted use, distribution, and reproduction in any medium, provided the original work is properly cited. 
nodes is of paramount importance. Also, ad hoc nodes are likely to be equipped with a smaller number of radio interfaces than mesh routers in wireless mesh networks, hence utilizing available channels with a limited number of radio interfaces is a key design goal. Considering these two central points, we take a hybrid channel assignmentbased approach and deal with fair rate control problems in such a context.

In both wireless mesh networks and wireless ad hoc networks which are typically based on commodity IEEE 802.11 radio nodes, interference from external devices such as residential access points or Bluetooth devices in industrial, scientific, and medical (ISM) bands that do not belong to those networks can severely affect the performance of those networks [8]. Such external devices are referred to as primary nodes (PNs). Recently, cognitive radio platforms such as those in [9-11] have been applied to wireless mesh networks to avoid interference from PNs where cognitive mesh routers coexist efficiently with the PNs in ISM bands by balancing channel load of the PNs and cognitive mesh routers. Cognitive mechanisms in the ad hoc network context are equally important, but they have not been studied much in the literature.

In this paper, we attempt, to the best of our knowledge, to conduct the first study of fair rate control problems in hybrid channel assignment-based multi-channel wireless ad hoc networks in the cognitive network context and seek for a distributed solution in the ad hoc context. Allocating optimal rates to nodes in a fair manner should jointly consider all the protocol stacks from scheduling at the medium access control (MAC) layer to channel assignment at the link layer, routing at the network layer, and rate control at the transport layer. It is essentially a very complex problem, but we can make an approximation approach to the problem by first using specific routing algorithms such as those in [5,9] and cognitive channel assignment and then narrowing it down into cross-layer rate control at the transport layer and scheduling at the MAC layer. We present a distributed cognitive channel assignment algorithm for ad hoc networks. To solve cross-layer rate control and scheduling problems we exploit a canonical form of network utility maximization framework specifically tailored to our cognitive ad hoc network context. In so doing, we discovered two new constraints inherent to hybrid channel assignment and primary node interference, i.e., interference constraint and per-interface constraint. We demonstrate that both constraints are unique and significant in multi-channel networks as opposed to legacy single-channel networks or static channel assignment-based multi-channel networks. We develop a price-based fair rate control algorithm that achieves fast convergence by simultaneously updating source rates at the transport layer and prices for both constraints at the link layer. In a simulation study over a grid topology, we provide a detailed analysis of the constraints and the convergence property of source rates. In the context of cognitive networks, it is important that congestion control be adaptive to spectrum usage changes of primary nodes. We show how our proposed algorithm achieves the convergence in response to a change in primary user interference. We also conduct a comparison study with a generic class of existing static channel assignment-based works.

The remainder of the paper is as follows. In Section 2, we review previous works in the literature that are the most relevant to our work. In Section 3, we design a hybrid channel assignment-based multi-channel wireless ad hoc network which maintains network connectivity while being cognitive to interference from PNs. In Section 4, we develop a system model unique to our specific fair rate control problem. In Section 5, we devise a distributed solution to the model. In Section 6, we present and analyze the performance of the proposed fair rate control algorithm based on cognitive hybrid channel assignment. Lastly, in Section 7, we remark on major contributions and findings in this paper.

\section{Related work}

\subsection{Channel assignment}

Multi-channel wireless networks have been much studied in the context of both wireless mesh networks and ad hoc networks. Channel assignment has been an early focal point. Ramachandran et al. [3] proposed the use of static channel assignment for wireless mesh networks where each node can have more than two radios. They require a fixed interface operating on a common channel for the entire connectivity within a mesh network. Kyasanur et al. [4] first proposed hybrid channel assignment and routing algorithms where some interfaces are fixed for packet reception, whereas others are dynamically switchable for packet transmission. Yoon et al. proposed an enhanced hybrid channel assignment algorithm [5]. Our work is influenced by this generic class of hybrid channel assignment, but we propose an enhanced hybrid channel selection algorithm considering interference in the presence of primary nodes. We also deal with a fair rate control problem on top of the hybrid channel assignment problem and demonstrate that achievable rates with only two radio interfaces per node are higher than static channel assignment methods. The case of using two radios in the mesh network context was first made by Dhananjay et al. [12]. Unlike their focus on routing and channel assignment in the mesh network context, we primarily tackle fair rate control problems in the ad hoc network context. Franklin et al. [13] proposed reconfiguration algorithms for channel assignment and flow rate allocation in response to dynamic traffic demands, unlike most previous works dealing with the static traffic demand. Avallone 
et al. [14] considered a new set of flow traffic in adapting channel and rate assignment by minimizing the maximum total utilization, which resulted in the enhancement of throughput. Similar to this trend of adaptive channel assignment, we propose a long-term channel reselection method for the fixed receiving interface with careful consideration not only of in-network node population but also of interference from out-of-network primary nodes.

\subsection{Rate control in multi-channel wireless networks}

Turning to the rate control perspective of multi-channel wireless networks, network utility maximization (NUM) framework has also been applied to some works in the context of mesh networks rather than ad hoc networks [6,7]. Giannoulis et al. [6] first proposed novel NUMbased congestion control and channel assignment algorithms in multi-channel wireless mesh networks (WMNs) using a clique-based interference model. Owing to the complexity of allowing all possible multiple paths for a single flow and calculating all cliques existent in a network, the proposed algorithms may not be computationally practical and thereby posing heavy overhead in distributed implementation. Tang et al. [7] comprehensively considered various fairness criteria for joint rate control and routing problems in multi-channel WMNs, e.g., throughput maximization, max-min fairness, and proportional fairness. The linear programming (LP)- and convex programming $(\mathrm{CP})$-based solutions are uniquely centralized, which assumes the existence of a central entity. Unlike previous works, our work focuses on rate control and channel assignment in the ad hoc network context, and thus, maintaining network connectivity in dynamic network changes leads us to distributed hybrid channel assignment-based solutions implementable with lesser overhead.

\subsection{Cognitive radio networks}

Recently, cognitive radio networks have drawn ongoing extensive interests. Shi et al. [15] for the first time formulated a cross-layer joint power control, frequency band scheduling, and routing problem using a physical interference model in a cognitive radio context into a mixed integer nonlinear program and proposed a centralized heuristic algorithm with relaxation technologies. Tang et al. [16] for the first time considered a max-min fair resource allocation in cognitive radio networks. They formulated a joint routing, scheduling, and spectrum allocation and proposed a centralized linear programming-based solution. Unlike the above-mentioned previous works, our focal point is the design of distributed fairness mechanism in the ad hoc context with additional accommodation of a cognitive mechanism to cope with interference on the ISM bands. Presti et al. [17] consider cross-layer flow rate, routing, and medium access control in a cognitive radio network context and, for the first time, derived a fully distributed solution. Unlike a general cognitive radio network solution which exploits spectrum holes on licensed frequency bands and assumes all the nodes equipped with all radio interfaces for all available frequency bands [17], our target network is IEEE 802.11-based multi-channel ad hoc networks on the unlicensed ISM bands, and we do seek a cost-effective solution with a lesser number of radio interfaces than available channels.

\section{Cognitive multi-channel wireless ad hoc network}

\subsection{Hybrid channel assignment-based network connectivity}

Our target is the hybrid channel assignment-based multichannel wireless ad hoc network which can coexist with primary nodes in a cognitive manner as in Figure 1. Each ad hoc node is equipped with only two radio interfaces based on the hybrid channel assignment to exploit spectral diversity of multiple available channels (e.g., five channels in Figure 1) and to increase the capacity $[4,5]$. One is a fixed interface for receive operation only on a dedicated channel, while the other is a switchable interface for transmit operation by changing its operating channel to that of an intended destination node. The color and number near the fixed receiving interface of each node in Figure 1 represent an operating channel. The number near each primary node indicates the channel impacted by its interference in Figure 1. The channel used for the fixed interface changes on a long time scale to cope with changes in a topology. Dynamic change of channels by the switchable interface in fact creates dynamic links on different channels to different neighbor nodes. Such a hybrid channel assignment in multi-channel approaches achieves high capacity while maintaining connectivity with neighbor ad hoc nodes using the switchable transmitting interface. We will show later that the minimal set of two interfaces works very well even in the presence of more than two available channels. The present switching latency can be reduced to small amounts, e.g., $100 \mu \mathrm{s}$ to $1 \mathrm{~ms}$ [4], but efficient switching scheduling to the channels of neighbor ad hoc nodes would help reduce the total overhead. Primary nodes in our scenario are composed of devices operating in the unlicensed ISM bands such as residential wireless local area network (WLAN) access points and Bluetooth devices. Limited to the ISM bands, we assume that PNs in our scenario have relatively short radio ranges compared to, for example, TV signals which are of most concern in cognitive radio-based solutions.

\subsection{Cognitive radio mechanism: spectrum sensing and channel selection}

In order to make an optimal channel assignment for the fixed receiving interface, each ad hoc node senses 


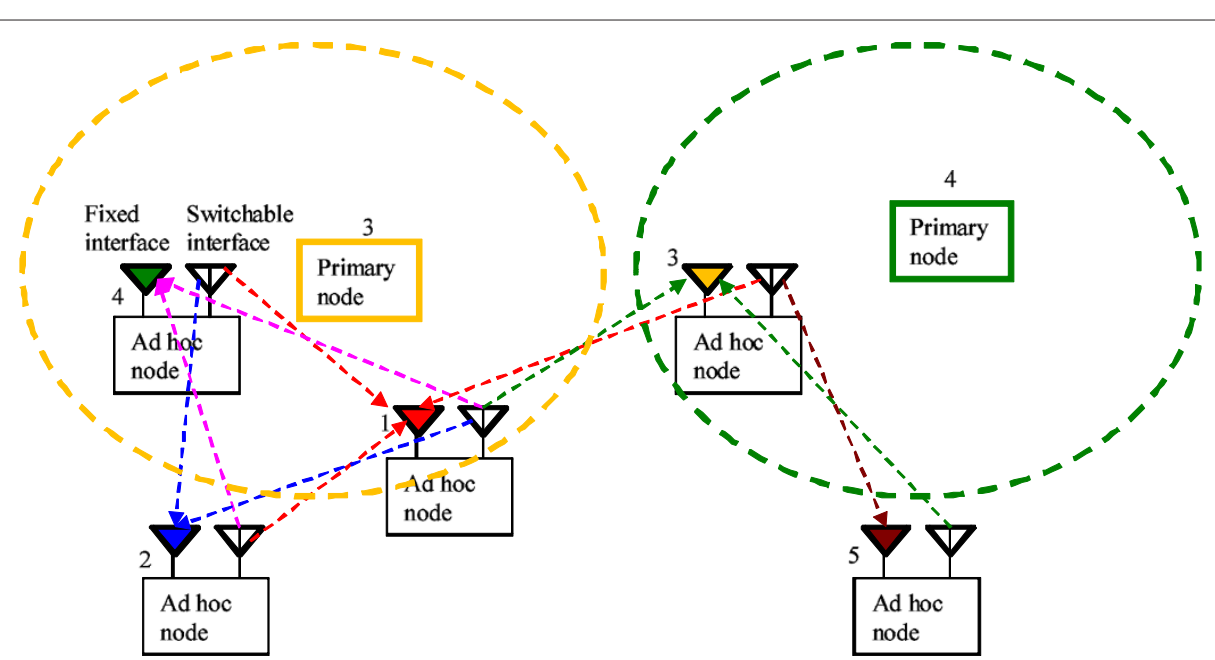

Figure $1 \mathrm{~A}$ hybrid channel assignment-based ad hoc network in the presence of primary nodes.

periodically a radio spectrum on both interfaces and estimates PN traffic workloads. Based on the sensed information, it selects a channel that is less occupied by external traffic caused by PNs for the fixed receiving interface. For spectrum sensing, it periodically samples the energy level on given bands. By following radio resource measurements (e.g., 802.11k) based on channel occupancy and/or interference [18], an ad hoc node can then estimate PN traffic workload on each channel. In a similar way as the mesh network case $[9,10]$, such PN traffic can be captured by a semi-Markov model with two states, that is, a busy state and an idle state. Idle time, $T_{\text {idle, and busy }}$ duration, $T_{\text {busy }}$, have cumulative distribution functions (CDFs) of two exponential random variables with rate $\lambda$ and $\mu$, respectively, given by $P\left[T_{\text {idle }} \leq t\right]=1-e^{-\lambda t}$ and $P\left[T_{\text {busy }} \leq t\right]=1-e^{-\mu t}$. The PN traffic workload $\left(\omega=\frac{T_{\text {busy }}}{T_{\text {busy }}+T_{\text {idle }}}\right)$ can then be estimated by sampling channel status (busy or idle) during a given sensing window, where we require that all ad hoc nodes do not send any traffic in order to identify external PN traffic. Synchronization for the sensing window duration can easily be achieved using methods similar to those in [19]. The longer the sensing window, the better is the workload estimation but the more overhead will the sensing bring up. Collaborative sensing that exchanges measured channel information among ad hoc nodes may help reduce the overhead.

Based on the measured workload of external PN traffic $\omega$ and the physical data rate of the radio interface $r_{0}$ (e.g., $72 \mathrm{Mbps}$ for IEEE $802.11 \mathrm{n}$ of $20-\mathrm{MHz}$ bandwidth with a single antenna [20]), we can approximate the expected capacity $r_{i}$ for channel $i$ as follows:

$$
r_{i}=r_{0} \times(1-\omega)
$$

Then, each node $n$ can approximate the channel capacity per node $r_{i}(n)$ as follows:

$$
r_{i}(n)=\frac{r_{i}}{N_{i}(n)}
$$

where $N_{i}(n)$ is the number of transmitting ad hoc nodes tuning to channel $i$ within the interference range of $n$ assuming fair use of available capacity among the transmitting nodes. Combining the information about PN workload with the estimation of the number of neighbor nodes, node $n$ can consider both internal interference (caused by other ad hoc nodes) and external interference (caused by PNs) in selecting its own channel. More specifically, each node $n$ selects a channel $j$ for its fixed interface such that

$$
j=\arg \max _{i} r_{i}(n) .
$$

Then, it tunes its receiving interface to channel $j$. It notifies its own channel and neighbors' channel selection information to other neighbors in a control message.

\section{System modeling}

\subsection{Target network}

We consider a multi-channel wireless ad hoc network that adopts hybrid channel assignment in which one interface is fixed to a channel for receive operation and the other interface is switchable to various channels for transmission operation $[4,5,9]$. With such hybrid channel assignment, our target network is therefore modeled as a directed graph $G=(V, L)$ where $L$ is defined as a set of directional links representing subflows. Thus, for two nodes $i$ and $j \in V$, there are two links $(i \rightarrow j)$ and $(j \rightarrow i)$. 
In addition, subflow $(i \rightarrow j)$ is highly likely to operate on a different channel to subflow $(j \rightarrow i$ ) since neighbor nodes $i$ and $j$ would not choose the same channel for its fixed receiving interface. Note that even when the channel of $i$ 's receiving interface is different from that of $j$ 's receiving interface, the network topology is maintained because $i$ 's switchable transmitting interface is tuned to the channel of $j$ 's receiving interface, and similarly, $j$ 's switchable transmitting interface is tuned to the channel of $i$ 's receiving interface. $S$ is a set of flows with a source and a destination. $x_{s}$ is the rate for flow $s \in S$. Let $U_{s}(\cdot)$ denote a utility function for flow $s$. We assume single-path routing here since multi-path routing is rarely used in real networks and potentially makes the overall system complex, e.g., packet reordering problem [21]. Also, the use of multi-path routing inherently requires double convergence loops for fair rate convergence [22] which makes convergence time much longer. An algorithm for routing is independent of channel assignment methods, e.g., shortest path routing should be enough in multi-channel networks to which simplifies the routing problem without loss of performance as shown in [5]. Several time slots should be scheduled for quiet periods to sense channel workload, which can reduce the link capacity to less than 1. In terms of throughput for long duration, however, the time slots for sensing overhead can be ignored compared to the ones for data transmissions.

\subsection{Problem formulation}

The problem of maximizing network utility can be formulated into matrix form as follows:

$$
\max \sum_{s} U_{s}\left(x_{s}\right)
$$

subject to $\quad(\mathbf{I} \otimes \mathbf{A}) \mathbf{R} \mathbf{x} \preceq \eta$

$$
\begin{aligned}
& \text { VRx } \preceq 1 \\
& \mathbf{0} \preceq \mathbf{x} .
\end{aligned}
$$

Here, $\mathbf{I}$ is an $|L|$ by $|L|$ matrix in which $I_{i j}=1$ if link $j$ is within the interference range of link $i$; otherwise, $I_{i j}=0$. $\mathbf{I}$ is determined by a given network topology and the interference model we use in this paper. The interference model imposes the interference constraint which will be explained in the next paragraph. Note that I implies potential interference only, and actual interference depends also on channel assignment. Link $i$ suffers from self-interference, i.e., $I_{i i}=1$ for each link $i$. $\mathbf{A}$ is an $|L|$ by $|L|$ dimensional channel assignment matrix in which $A_{i j}=1$ if link $j$ is on the same channel as link $i$; otherwise, $A_{i j}=0$. Operator $\otimes$ is defined such that $(I \otimes A)_{i j}=I_{i j} \cdot A_{i j}$ for two matrices $\mathbf{I}$ and $\mathbf{A}$ of the same dimension. Thus, two links $i$ and $j$ interfere with each other if they are within the interference range and operate on the same channel.
Note that operator $\otimes$ is different from matrix multiplication. $\mathbf{R}$ is an $|L|$ by $|S|$ routing matrix in which $R_{i j}=1$ if flow $j$ passes link $i$; otherwise, $R_{i j}=0 . \mathbf{x}$ is a flow rate vector with $0 \leq x_{s}, \forall s \in S$. $\mathbf{V}$ is a $|V|$ by $|L|$ matrix where $V_{i j}=1$ if link $j$ is incident from node $i$; otherwise, $V_{i j}=0.1$ is a vector with all $1 . \eta$ is a vector with $\eta_{l}=1-\omega_{l}$ where $\omega_{l}$ is the channel workload on the channel of link $l$ which is estimated by the proposed cognitive mechanism in Section 3.2. In our target cognitive multichannel network, node channel assignment for the fixed receiving interface is completed prior to a fair rate control algorithm, and thus, $\omega_{l}$ is easily obtained from spectrum sensing of the pre-assigned channel by the destination of link $l$. That is, the destination node of link $l$ dynamically senses the available channel bandwidth $\eta_{l}$ in a cognitive multi-channel network.

With the set of definitions above, the first constraint in Equation 4 implies that the sum of time fraction used by a link $l$ and its potentially interfering links cannot exceed $\eta_{l}$. The constraint, called interference constraint as in [2], is well known to be a sufficient condition for feasible subflow schedules [23]. The constraint belongs to the class of IEEE 802.11 protocol interference models and is hence simple to implement in a distributed way in ad hoc networks. In the ad hoc network context, we do not use clique-based interference models, e.g., [6], which may have significant computation overheads in ad hoc networks and have to reduce a clique capacity sufficient enough for feasible subflow schedules. Signal-to-interference noise ratio (SINR)-based physical interference models [24] may be more accurately applied to multi-radio multi-channel networks, but the computational complexity of calculating SINR in the ad hoc environment may be significantly high.

The second constraint in Equation 4 means that time fraction used by the outgoing interface of a node cannot exceed 1 . Note that this is a new constraint which we first identify to be unique in hybrid channel assignmentbased multi-channel networks. We call it per-interface constraint, and it is tight and more exact in comparison with the per-node constraint where the usage of channel time is bounded by the total number of radios per node [2]. Note also that the interference constraint does not subsume the per-interface constraint in multi-channel networks as opposed to legacy single-channel models. We will find this argument to hold true later in an example of multi-channel topology in Section 6.1.

Matrices $\mathbf{I}$ and $\mathbf{V}$ are determined by a given network topology and a given interference range. An interference range depends on a sender's transmission power and distance from an interfering source. It is larger than a transmission range, and we typically use an interference range of 2 to 4 times a transmission range. Since we take a decoupling approach that $\mathbf{R}$ is predetermined by some routing algorithms and $\mathbf{A}$ is determined by our hybrid 
channel selection scheme at first, what remains is the problem of optimizing the flow source rate $\mathbf{x}$ at the transport layer and transmission scheduling at the MAC/link layer in a cross-layer manner to achieve fairness. Note that a cognitive routing algorithm such as that in $[25,26]$, not a simple multi-channel routing, must be adopted in the presence of PNs. In Section 5, we will devise a distributed solution to the above-mentioned optimization problem.

\section{Distributed solution}

\subsection{Dual problem}

We have the Lagrangian by introducing two Lagrange multipliers: $\lambda$ which is associated with links by the first constraint in Equation 4 and $\boldsymbol{\mu}$ which is associated with nodes by the second constraint in Equation 4:

$$
\begin{aligned}
L(\mathbf{x}, \lambda, \boldsymbol{\mu})= & \sum_{s} U_{s}\left(x_{s}\right)-\sum_{l} \lambda_{l}\left(\sum_{s}(I \otimes A) R_{l s} x_{s}-\eta_{l}\right) \\
& -\sum_{v} \mu_{v}\left(\sum_{s} V R_{v s} x_{s}-1\right) \\
= & \sum_{s}\left(U_{s}\left(x_{s}\right)-x_{s}\left(\sum_{l} \lambda_{l}(I \otimes A) R_{l s}+\sum_{v} \mu_{v} V R_{v s}\right)\right) \\
& +\sum_{l} \lambda_{l} \eta_{l}+\sum_{v} \mu_{v}
\end{aligned}
$$

The dual function is

$$
D(\boldsymbol{\lambda}, \boldsymbol{\mu})=\max _{\mathbf{x} \succeq \mathbf{0}} L(\mathbf{x}, \boldsymbol{\lambda}, \boldsymbol{\mu}),
$$

and the dual problem to Equation 4 is

$$
\min \quad D(\lambda, \mu)
$$

$$
\text { subject to } \quad \lambda \succeq \mathbf{0}, \boldsymbol{\mu} \succeq \mathbf{0} \text {. }
$$

With an assumption that utility functions $U_{s}$ are increasing and strictly concave, by duality theory, we can find the optimal to the primal problem (4) by solving the dual problem (7).

\subsection{Fair rate control algorithm}

We solve the dual problem (7) using the gradient projection method. Link prices $\lambda$ and interface prices $\boldsymbol{\mu}$ are updated by the following rules:

$$
\begin{aligned}
\lambda_{l}(t+1) & =\left[\lambda_{l}(t)-\gamma \frac{\partial D(\lambda(t), \boldsymbol{\mu}(t))}{\partial \lambda_{l}}\right]^{+} \\
& =\left[\lambda_{l}(t)-\gamma\left(\eta_{l}-\sum_{s} x_{s}(\lambda(t), \mu(t))(I \otimes A) R_{l s}\right)\right]^{+}
\end{aligned}
$$

$$
\begin{aligned}
\mu_{\nu}(t+1) & =\left[\mu_{\nu}(t)-\gamma \frac{\partial D(\lambda(t), \boldsymbol{\mu}(t))}{\partial \mu_{l}}\right]^{+} \\
& =\left[\mu_{\nu}(t)-\gamma\left(1-\sum_{s} x_{s}(\lambda(t), \boldsymbol{\mu}(t)) V R_{v s}\right)\right]^{+}
\end{aligned}
$$

where $\gamma$ is a step size, and $[z]^{+}=\max \{z, 0\}$. Each node $v$ feeds back $\mu_{\nu}$ and $\lambda_{l}$ where it is the destination of link $l$ to sources $s$ where $R_{l s}=1$.

Since $U_{s}$ is differentiable, each source $s$ determines $x_{s}$ by the following rule [27]:

$$
\begin{aligned}
x_{s}(t+1)= & {\left[x_{s}(t)+\gamma x_{s}(t)\left(U_{s}^{\prime}\left(x_{s}(t)\right)-\left(\sum_{l} \lambda_{l}(I \otimes A) R_{l s}\right.\right.\right.} \\
& \left.\left.\left.+\sum_{v} \mu_{v} V R_{v s}\right)\right)\right]^{+} .
\end{aligned}
$$

Then, source $s$ will inform all nodes on its route of newly updated $x_{s}$ value. Note that the proposed algorithm is a cross-layer primal-dual algorithm as link prices and interface prices are actively updated at the link layer and source rates are actively updated at the transport layer. It is also a distributed algorithm with local information exchange as link prices and interface prices are known to involve source nodes only, and source rates are known to nodes over the route from each source only, but not to the entire network. The proposed algorithm can be implemented in real time since source rates and shadow prices are explicitly exchanged and accordingly updated at each iteration.

\section{Performance evaluation}

\subsection{Convergence property}

For the verification of the proposed distributed fair rate control algorithm, we first use a $4 \times 4$ grid topology in Figure 2 which is on an appropriate scale for in-depth analysis. We assume that five nonoverlapping channels are available in the wireless network. Note that, typically, three channels can be used without interference among a total of 13 channels of IEEE 802.11a WLAN standards $[4,28]$. For IEEE 802.11n, four orthogonal channels of $20 \mathrm{MHz}$ are allowed, and for IEEE 802.11g, three channels of $22 \mathrm{MHz}$ are available. The operating channels of the receiving interface of each node are assigned using the proposed hybrid channel selection scheme as indicated in the parenthesis of each node. A PN is using channel 4 and thus interfering with link $H$ to $F$. Suppose that the $\mathrm{PN}$ workload is 0.25 . A total of four flows is considered, and we assume that routing is determined as shown in the figure. 


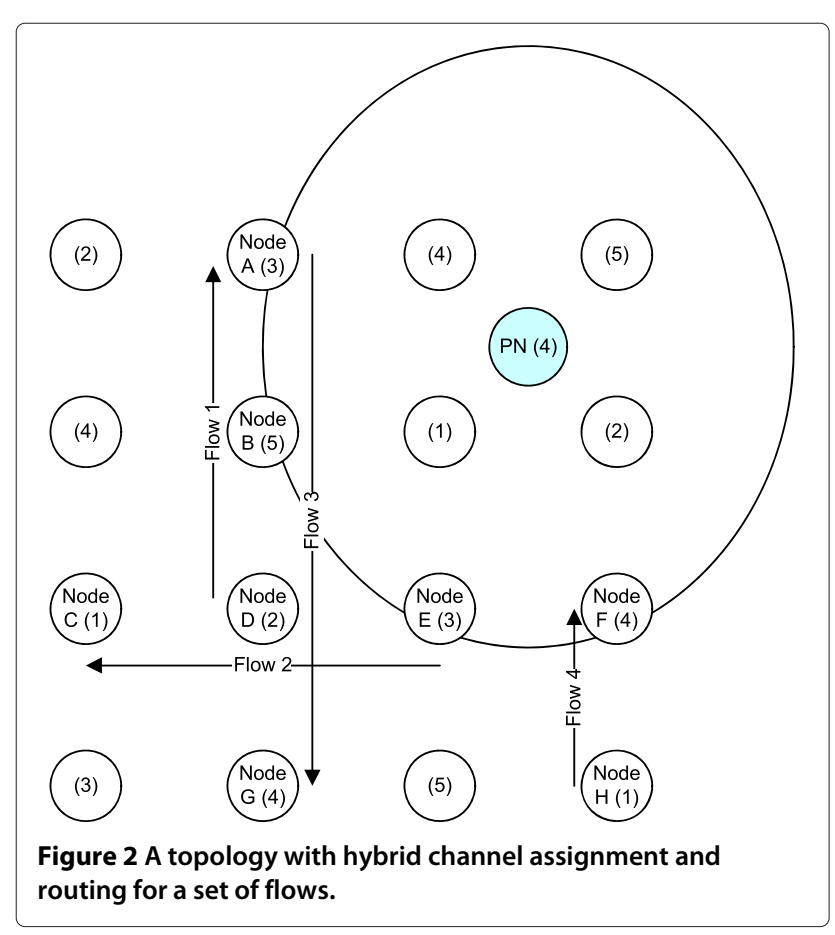

We use the uniform utility function $U_{s}\left(x_{s}\right)=\log \left(x_{s}\right)$ to achieve the proportional fairness objective, and the obtained proportional fair flow rates are normalized in Table 1. Figure 3 plots the rates of each flow as the distributed update of the interface price of each node, and each link price is iterated. The step size $\gamma$ is set to 0.1 , and the optimal rates are achieved upon 172 iterations. The optimal network utility at equilibrium is -1.7519 . Note that both the interference constraint and the per-interface constraint are significant. For example, the per-interface constraint at node $D$ determines the rates of flow $x_{1}$, $x_{2}$, and $x_{3}$, and the interference constraint on channel 4 in the given cognitive multi-channel network determines the rates of flow $x_{3}$ and $x_{4}$. From the result, we demonstrate that the proposed solution achieves the proportional fairness by taking into account both the interference constraint and the per-interface constraint. For the purpose of this article, we report several numerical results demonstrating convergence although the formal proof of stability can be done following the canonical NUM framework [27].

Figure 4 plots the convergence of link prices and interface prices. Initially, all the link prices and all the switchable transmitting interface prices are set to 1 . At equilibrium, the price for link $H$ to $F$ converges to 1.899 ,

Table 1 Proportional fair rates

\begin{tabular}{lcccc}
\hline & Flow 1 & Flow 2 & Flow 3 & Flow 4 \\
\hline Normalized flow rate & 0.388 & 0.388 & 0.223 & 0.527 \\
\hline
\end{tabular}

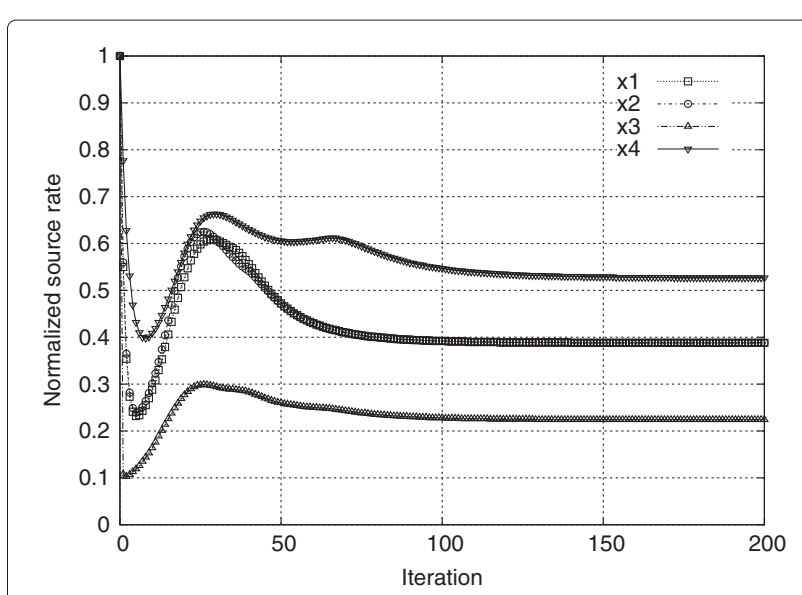

Figure 3 The convergence of flow source rates.

while the price for all other links is reduced to 0 . This implies that link $H$ to $F$ is the limiting factor to flow 3 and flow 4 . At equilibrium, the interface prices for node $D$ converges to 2.576, while the interface price for all other nodes is reduced to 0 . This implies that the switchable transmitting interface of node $D$ is the limiting factor to flow 1 , flow 2 , and flow 3 . In this example, we can argue that both interference constraint and per-interface constraint and their corresponding link prices and interface prices, respectively, play crucial roles in the fair rate control of multi-radio multi-channel wireless ad hoc networks.

\subsection{Cognitive radio mechanism: adaptation to dynamic channel usage by PN}

We study how the proposed congestion control algorithm adapts to dynamic channel usage by the PN. Let us consider the equilibrium state in the above scenario. Suppose that the PN does not use the wireless channel 4 any longer, and hence, the cognitive mesh node $F$ detects this change in channel 4. Node $F$ notifies node $H$ of the change, and node $H$ subsequently modifies its $\eta_{l}$ variable for link ( $H$ to $F$ ) from the current value 0.25 to a new value 0 . Note that in all these steps, only the local information ( $\eta_{l}$ for link $H$ to $F$ ) is manipulated in a distributed way (between nodes $H$ and $F$ ) without involvement of other nodes. As in this case, the proposed algorithm is local and distributed in adaptation to dynamic channel usage in cognitive multi-channel wireless networks.

Table 2 shows the updated proportional fair flow rates in response to the change in the $\mathrm{PN}$ channel usage $\left(w_{l}: 0.25 \rightarrow 0\right)$. The maximal network utility is now increased to -1.5687 , thanks to the proposed cognitive radio mechanism. Figure 5 confirms that the converged rates obtained by the proposed algorithm are the same as those that achieve maximal network utility. The proposed algorithm completes convergence with about 60 iterations. We also plot the convergence of price variables 


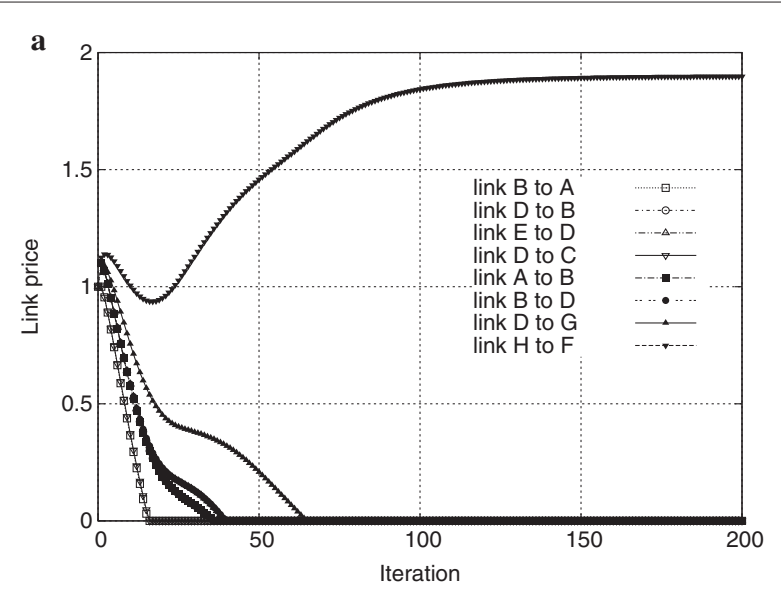

b

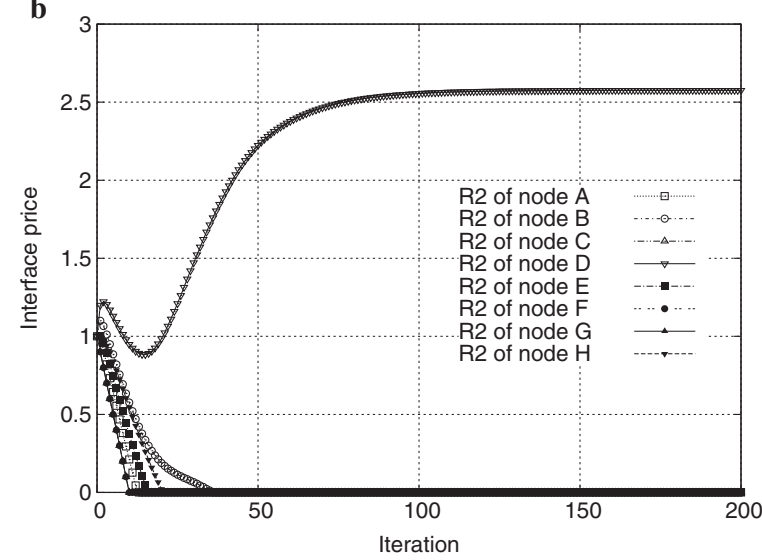

Figure 4 The convergence of (a) link prices and (b) interface prices. R2 means the switchable radio interface used for transmission.

in response to the PN channel usage change in Figure 6. We can observe the convergence of the limiting resources, i.e., link from $H$ to $F$, and it confirms that the converged rates obtained above by the proposed algorithm are the ones that achieve maximal network utility.

\subsection{Comparison with existing works}

In this section, we present extensive simulation results on random topologies. Through random topology simulations, we will demonstrate the convergence property of the proposed fair rate control algorithm in more realistic random topologies. Such simulations will also serve as a comparison of our hybrid channel assignment-based fair rate control against a generic class of static channel assignment-based rate control methods in the literature $[6,29]$. We will focus on convergence properties and total

Table 2 Updated proportional fair rates

\begin{tabular}{lcccc}
\hline & Flow $\mathbf{1}$ & Flow 2 & Flow $\mathbf{3}$ & Flow $\mathbf{4}$ \\
\hline Normalized flow rate & 0.375 & 0.375 & 0.25 & 0.75 \\
\hline
\end{tabular}

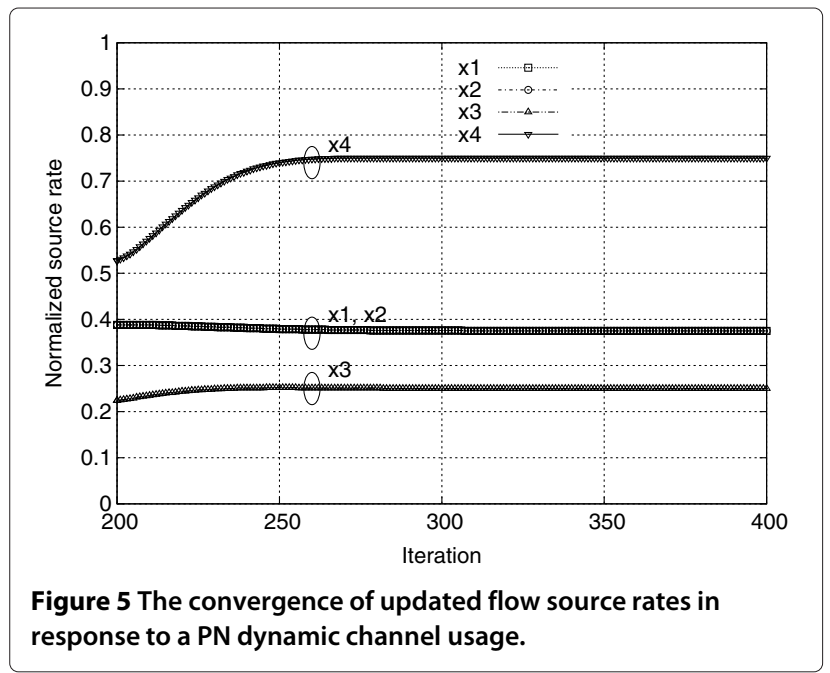

network utilities. An example of random topologies generated in the simulator is depicted in Figure 7 where $100 \mathrm{ad}$ hoc nodes are placed. The color of each node represents the operating channel of its fixed receiving interface. Among them, we select 30 sources and 30 destinations to
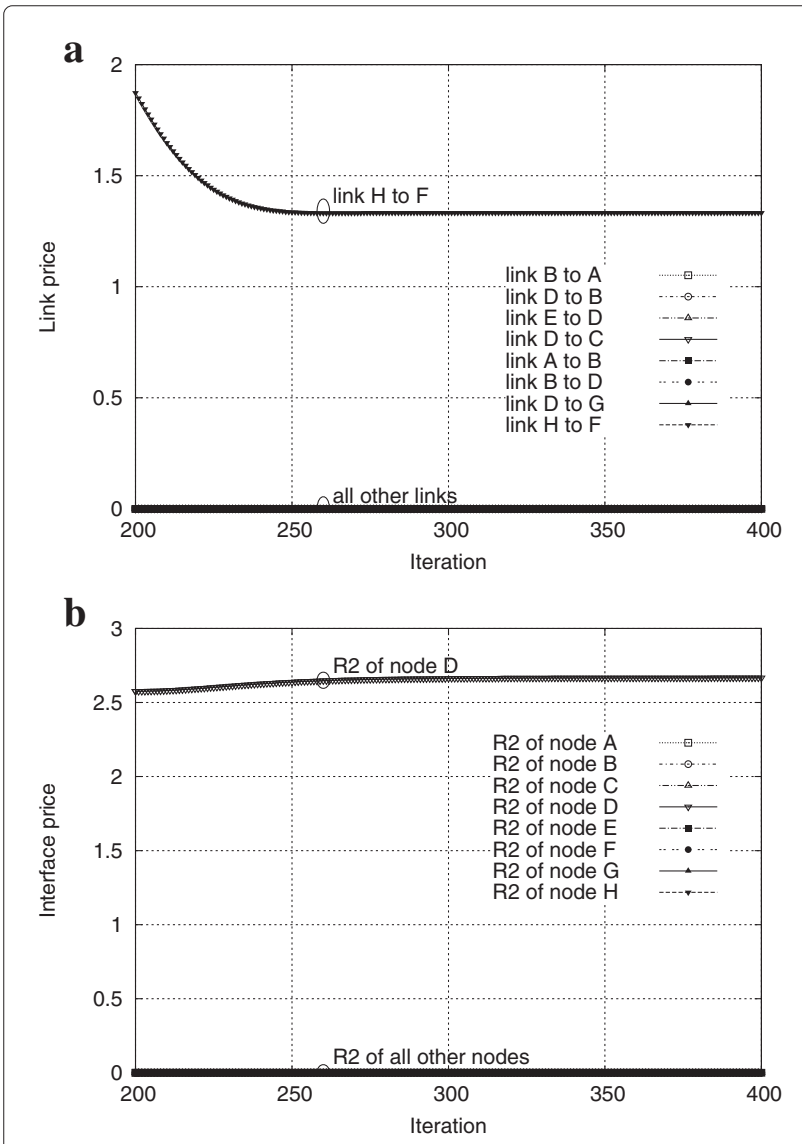

Figure 6 The convergence of updated resource prices in response to a PN dynamic channel usage. R2 means the switchable radio interface used for transmission. (a) Updated link prices. (b) Updated interface prices. 
make a total of 30 flows. To consider proportional fairness, we use the uniform utility function $U_{s}\left(x_{s}\right)=\log \left(x_{s}\right)$ for each flow $s$. The interference range is set to four hops to reflect more aggregate interference from concurrent transmission in such dense topologies. The number of available channels are set to four by considering the current IEEE 802.11n technology where four orthogonal channels are defined each with 20-MHz bandwidth [20].

In Figure 8, we illustrate and compare the convergence property. Our proposed algorithm uses two radio interfaces only, whereas we vary the number of radio interfaces between two and four for static channel assignment methods. Our proposed algorithm shows convergence while achieving the highest total network utility of -93.305 . The static channel assignment methods also achieve proportional fairness but with less values of the network utility, e.g., -113.429 for two radios, -101.765 for three radios, and -95.845 for four radios. Having a higher network utility with two radios only is mainly attributed to the inherent channel diversity accomplished by hybrid channel assignment.

Figure 9 illustrates and compares the total network utility of all 30 flows for each different random topology. The proposed algorithm achieves far higher network utility than the static channel assignment methods with the same two radios and also three radios. It also performs comparably to the static channel assignment with four radios. In summary, the proposed algorithm is proven to be remarkably cost-effective in utilizing multiple available channels in ad hoc networks.

Figure 10 illustrates the cumulative distribution functions for flow rates in each case. Looking at the distribution of flow rates, we can figure out that the proposed fair rate control algorithm based on hybrid channel assignment allocates more resources efficiently to individual flows. We can note striking differences in individual flow rates when compared to the static channel assignment methods with two or three radios. Compared with the static channel assignment method with four radios, our algorithm has less number of exceedingly high individual flows and less number of low individual flows, thereby resulting in more balance among individual flows with higher total network utility.

\section{Conclusions}

In this paper, we dealt with a new cross-layer fair rate control problem in emerging multi-channel cognitive wireless ad hoc networks. Our work is based on hybrid channel assignment which provides good connectivity for wireless ad hoc networks, and we formulated it into a form of network utility maximization. We devised a distributed fair rate control algorithm that solves the formulated model

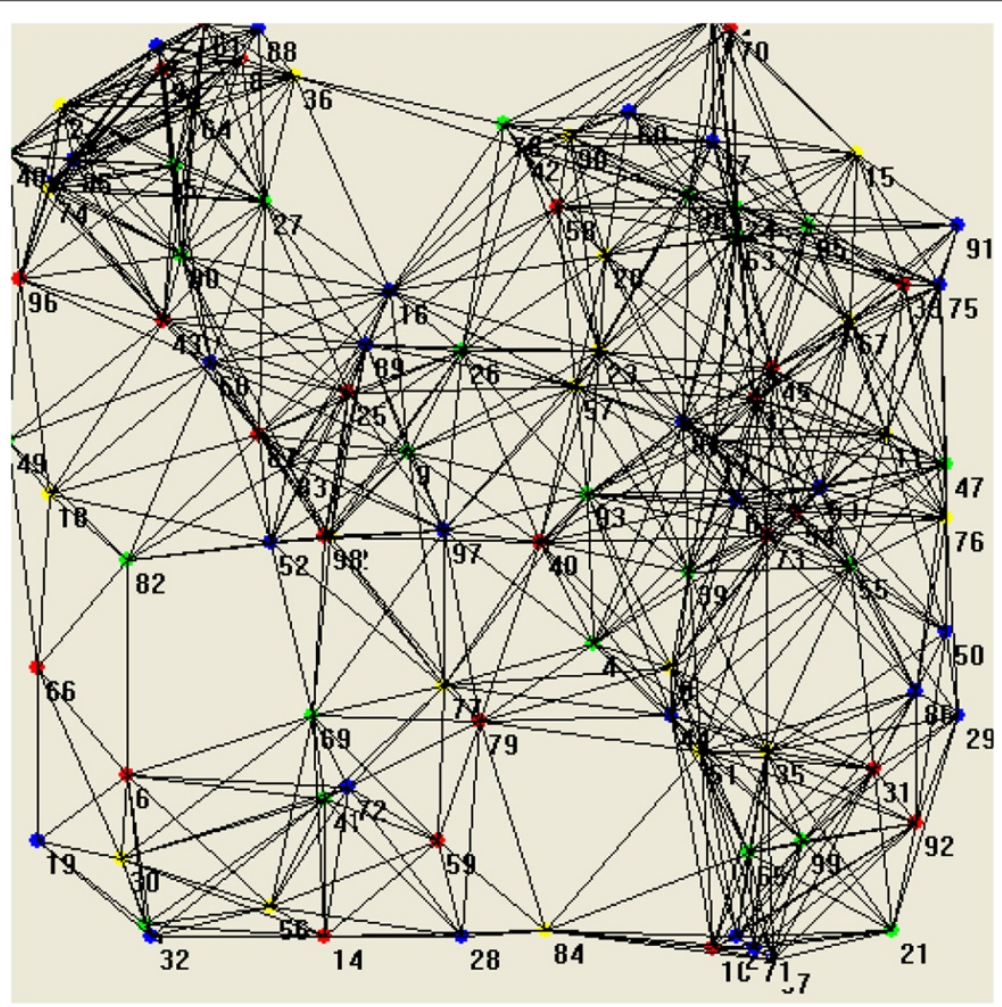

Figure 7 An example of random topology. 

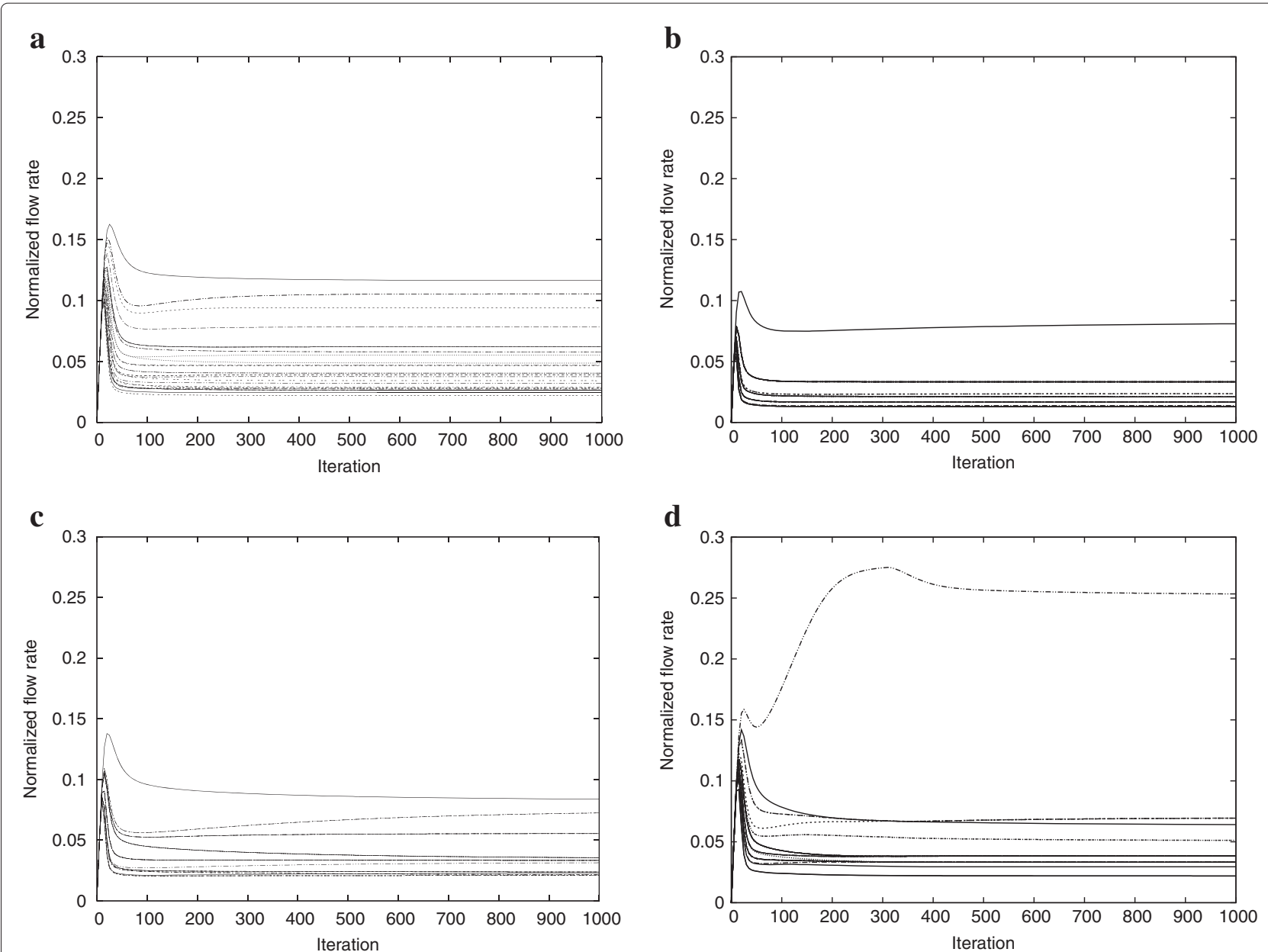

Figure 8 The comparison of convergence behaviors in the random topology in Figure 7 (topology ID 3). (a) Our algorithm (using two radios). (b) Static assignment with two radios. (c) Static assignment with three radios. (d) Static assignment with four radios.

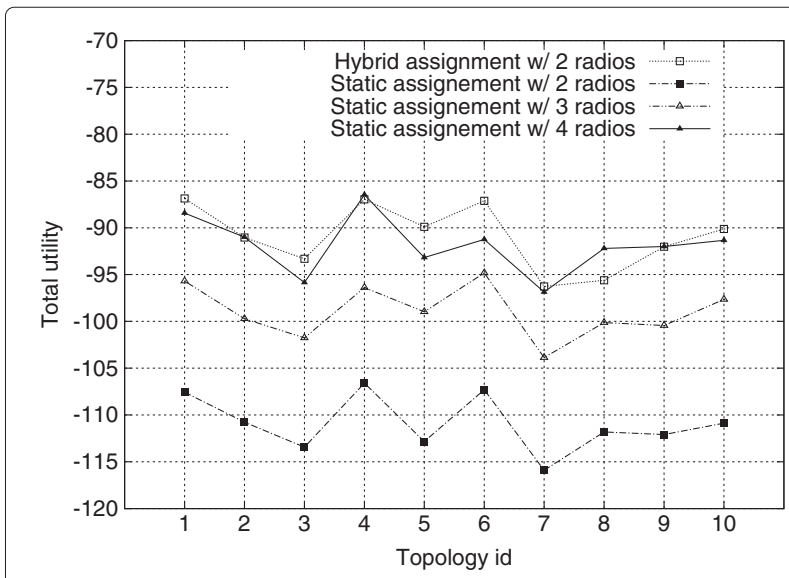

Figure 9 The comparison of the total utility for different random topologies.

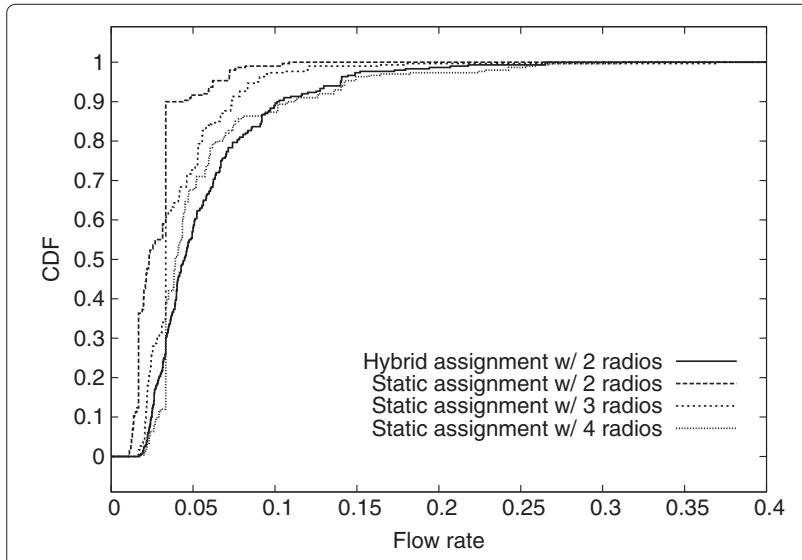

Figure 10 The comparison of cumulative distribution functions for normalized flow rates (for all ten topologies). 
and evaluated it in terms of fairness, convergence, and total network utility in both grid and more realistic random topologies.

We summarize the main findings of the paper. Firstly, in the formulated NUM-based system model, hybrid channel assignment has imposed unique constraints. That is, in addition to interference constraint which was prevalent in multi-channel network settings, we discovered the new per-interface constraint particularly relevant to hybrid channel assignment. There has been no work on formulating rate control problems in the context of hybrid channel assignment, and we demonstrated that previous per-node constraint cannot exactly model hybrid channel assignment-based networks. Secondly, in relation to the above finding, we discovered that fair rate control in hybrid channel assignment-based multi-channel networks should involve two different kinds of resources in radio resource allocation. In addition to the wellknown link prices which represent the cost of consuming the radio link capacity among mutually interfering transmissions, we developed the new interface prices which represent the cost of using the limited capacity of each transmitting interface. Thirdly, a cognitive radio mechanism is also devised and modeled into the problem formulation. We demonstrated that the proposed fair rate control algorithm is adaptive to the PN channel usage change in the cognitive context. Fourthly, we demonstrated that hybrid channel assignment-based fair rate control algorithm can achieve good connectivity and high network utility using only two radio interfaces. We presented a comparison study that articulates the strength of the proposed algorithm in terms of fairness and network utility against conventional static channel assignment methods with more number of radio interfaces. Fifthly and lastly, we conducted simulations on more realistic random topologies with a large number of nodes. Conventional works based on the NUM framework usually use small exemplary topologies with limited number of nodes. With our enhanced simulator, we were able to perform more extensive simulations in larger-scale realistic topologies, thereby presenting more substantial support for the convergence and fairness results.

Lastly, we note a direction for future works. Although channel switching delay is improved [4], we could further reduce the total cost induced by channel switching delay in our hybrid channel assignment-based solution. It is desirable if channel switch schedules for the transmitting interface can be contiguous as much as possible since switching to different receivers too often can result in time waste owing to channel switching [30]. Thus, an approach to schedule channel switching in a sophisticated time slot allocation manner might be an effective one.

\section{Competing interests}

The authors declare that they have no competing interests.

\section{Acknowledgements}

This work was supported by the National Research Foundation of Korea (NRF) grant funded by the Korea government (Ministry of Education) (no. 2012R1A1A2008478).

\section{Disclosure}

A preliminary version of the paper appeared in the Proceedings of the 7th International Conference on Ubiquitous Information Technologies and Applications 2012.

\section{Author details}

${ }^{1}$ Department of Electronic Engineering, Dong-A University, Hadan 2-dong, Saha-gu, Busan, South Korea. ${ }^{2}$ DMC R\&D Center, Samsung Electronics, Maetan 3-dong 416, Youngtong-gu, Suwon 443-732, South Korea.

Received: 17 September 2013 Accepted: 13 January 2014

Published: 17 January 2014

\section{References}

1. M Alicherry, R Bhatia, LE Li, Joint channel assignment and routing for throughput optimization in multi-radio wireless mesh networks, in ACM Mobicom'05, August 2005 (ACM, New York, 2005), pp. 58-72

2. M Kodialam, T Nandagopal, Characterizing the capacity region in multi-radio multi-channel wireless mesh networks, in ACM Mobicom'05, August 2005 (ACM, New York, 2005), pp. 73-87

3. KRamachandran, E Belding, K Almeroth, MM Buddhikot, Interference-aware channel assignment in multi-radio wireless mesh networks, in IEEE Infocom'06 (IEEE, Piscataway, 2006), pp. 1-12

4. P Kyasanur, NH Vaidya, Routing and link-layer protocols for multi-channel multi-interface ad hoc wireless networks. ACM SIGMOBILE MC2R. 10, 31-43 (2006)

5. W Yoon, NH Vaidya, A link layer protocol and link-state routing protocol suite for multi-channel ad hoc networks. Wireless Commun. Mobile Comput. 12, 85-98 (2012)

6. A Giannoulis, T Salonidis, E Knightly, Congestion control and channel assignment in multi-radio wireless mesh networks, in IEEESECON '08 (IEEE, Piscataway, 2008), pp. 350-358

7. J Tang, G Xue, W Zhang, Cross-layer design for end-to-end rate allocation in multi-radio wireless mesh networks. Wireless Netw. 15, 53-64 (2009)

8. D Gokhale, S Sen, K Chebrolu, B Raman, On the feasibility of the link abstraction in (rural) mesh networks, in IEEE Infocom'08 (IEEE, Piscataway, 2008), pp. 484-492

9. W Kim, AJ Kassler, MD Felice, M Gerla, L Bononi, Urban-X: a self-organizing cognitive wireless mesh network for dense city environments, in Wired/Wireless Internet Communications 2011 (Springer, Heidelberg, 2011), pp. 398-409

10. W Kim, A Kassler, MD Felice, M Gerla, Cognitive multi-radio mesh networks on ISM bands: a cross-layer architecture, in IEEE IPCCC'10, August 2010 (IEEE, Piscataway, 2010), pp. 34-41

11. W Kim, JS Park, Cross-layer scheduling for multi-users in cognitive multi-radio mesh networks. Wireless Commun. Mobile Comput. (2012). doi:10.1002/wcm.2256

12. A Dhananjay, H Zhang, J Li, L Subramanian, Practical, distributed channel assignment and routing in dual-radio mesh networks, in ACM Sigcomm'09, August 2009 (ACM, New York, 2009), pp. 99-110

13. A Franklin, A Balachandran, C Murthy, Online reconfiguration of channel assignment in multi-channel multi-radio wireless mesh networks. Comput. Commun. 35, 2004-2013 (2012)

14. S Avallone, GD Stasi, A Kassler, A traffic-aware channel and rate reassignment algorithm for wireless mesh networks. IEEE Trans. Mobile Comput. 12, 1335-1348 (2013)

15. Y Shi, YT Hou, H Zhou, SF Midkiff, Distributed cross-layer optimization for cognitive radio networks. IEEE Trans. Vehicular Technol. 59(8), 4058-4069 (2010)

16. J Tang, R Hincapié, G Xue, W Zhang, R Bustamante, Fair bandwidth allocation in wireless mesh networks with cognitive radios. IEEE Tras. Vechicular Technol. 59, 1487-1496 (2010) 
17. FL Presti, C Petrioli, Toward optimal cross-layer solutions for cognitive radio wireless networks, in IEEE CROWNCOM 2010 (IEEE, Piscataway, 2010), pp. 1-5

18. S Mangold, L Berlemann, IEEE $802.11 \mathrm{k}$ : improving confidence in radio resource measurements. IEEE PIMRC'05. 2, 1009-1013 (2005)

19. P Djukic, P Mohapatra, Soft-TDMAC: software TDMA-based MAC over commodity 802.11 hardware, in IEEE Infocom'09, April 2009 (IEEE, Piscataway, 2009), pp. 1836-1844

20. WG802.11 - Wireless LAN Working Group, in 802.11n-2009- IEEE Standard for Information technology - Local and metropolitan area networks - Specific requirements_Part 11: Wireless LAN Medium Access Control (MAC) and Physical Layer (PHY) Specifications Amendment 5: Enhancements for Higher Throughput. (IEEE, New York, 2009)

21. W Yoon, NH Vaidya, Routing exploiting multiple heterogeneous wireless interfaces: a TCP performance study. Comput. Commun. 33, 23-34 (2010)

22. DP Bertsekas, JN Tsitsiklis, Parallel and Distributed Computation: Numerical Methods. (Prentice-Hall, Upper Saddle River, 1989)

23. VSA Kumar, MV Marathe, S Parthasarathy, Algorithmic aspects of capacity in wireless networks. ACM SIGMETRICS'05. 33(1), 133-144 (2005)

24. Y Shi, YT Hou, J Liu, S Kompella, How to correctly use the protocol interference model for multi-hop wireless networks, in ACM MobiHoc '09, May 2009 (ACM, New York, 2009), pp. 239-248

25. AS Cacciapuoti, M Caleffi, L Paura, Reactive routing for mobile cognitive radio ad hoc networks. Ad Hoc Netw. 10, 803-815 (2012)

26. KR Chowdhury, IF Akyildiz, CRP: a routing protocol for cognitive radio ad hoc networks. IEEE J. Selected Areas Commun. 29, 794-804 (2011)

27. R Srikant, The Mathematics of Internet Congestion Control. (Walter de Gruyter GmbH, Birkhauser, Berlin, 2003)

28. V Angelakis, S Papadakis, V Siris, A Traganitis, Adjacent channel interference in 802.11a is harmful: testbed validation of a simple quantification model. IEEE Commun. Mag. 49, 160-166 (2011)

29. Z Ning, L Guo, Y Peng, X Wang, Joint scheduling and routing algorithm with load balancing in wireless mesh network. Comput. Electrical Eng. 32, 533-550 (2012)

30. CY Ku, YD Lin, SL Tsao, YC Lai, Utilizing multiple channels with fewer radios in wireless mesh networks. IEEE Trans. Vehicular Technol. 60, 263-275 (2011)

doi:10.1186/1687-1499-2014-11

Cite this article as: Yoon and Kim: Fair rate control for cognitive multi-channel wireless ad hoc networks. EURASIP Journal on Wireless Communications and Networking 2014 2014:11.

\section{Submit your manuscript to a SpringerOpen ${ }^{\mathcal{O}}$ journal and benefit from:}

- Convenient online submission

- Rigorous peer review

- Immediate publication on acceptance

- Open access: articles freely available online

- High visibility within the field

- Retaining the copyright to your article

Submit your next manuscript at $\boldsymbol{\wedge}$ springeropen.com 